\title{
APTITUD COMBINATORIA DEL RENDIMIENTO DE ANTOCIANINAS Y DE CARACTERÍSTICAS AGRONÓMICAS EN POBLACIONES NATIVAS DE MAÍZ PIGMENTADO
}

\author{
COMBINING ABILITY OF ANTHOCIANYNS YIELD AND AGRONOMIC TRAITS ON NATIVE \\ POPULATIONS OF PIGMENTED MAIZE
}

\author{
Edgar Espinosa Trujillo ${ }^{1}$, Ma. del Carmen Mendoza Castillo ${ }^{1 *}$, Fernando Castillo González ${ }^{1}$, Joaquín Ortiz \\ Cereceres $^{1} \uparrow$ y Adriana Delgado Alvarado ${ }^{2}$
}

${ }^{1}$ Instituto de Recursos Genéticos y Productividad, Colegio de Postgraduados, Campus Montecillo. Km 36.5, Carretera México-Texcoco. 56230, Montecillo, Texcoco, Estado de México. Tel 01 (595) 9520200 Ext. 1524. ${ }^{2}$ Postgrado en Estrategias para el Desarrollo Agrícola Regional, Colegio de Postgraduados, Campus Puebla. Km 125.5, Carretera México-Puebla. 72160, Puebla, Puebla.

* Autor para correspondencia (camen@colpos.mx)

\section{RESUMEN}

Los granos de maíz (Zea mays L.) rojos, azules y púrpuras contienen antocianinas de importancia en la prevención de enfermedades humanas. La selección por color de grano se considera relevante en la actualidad. Para lograr la definición de metodologías eficientes de selección conviene aplicar diseños genéticos que permitan analizar la contribución genética en la variación cuantitativa de las características de interés. Se usó un diseño genético para estimar los efectos de aptitud combinatoria general (ACG) y específica (ACE), maternos (EM) y recíprocos (ER) de la concentración de antocianinas por unidad de peso de grano $\left(C_{\mathrm{kg}}\right)$ y el rendimiento de antocianinas por hectárea $(\mathbf{R A h a})$. Se estableció un experimento de cruzas dialélicas entre cinco poblaciones nativas contrastantes en su color de grano, donde se midieron variables agronómicas. También se obtuvo la semilla $F_{2}$, con polinización controlada, para medir la concentración de antocianinas en el grano completo. Con base en los dos experimentos se calculó el rendimiento de antocianinas por planta (RApta), el RAha y la heterosis. Hubo semejanza entre progenitores en las variables agronómicas pero difirieron en su capacidad de acumular antocianinas. Los efectos de ACG fueron superiores en la mayoría de las características agronómicas, con respecto a los de ACE. En RApta hubo efectos significativos de ACG, ACE y ER, y fue la población Rojo-1 la de mayor ACG. Hubo heterosis entre cruzas para CAkg, siendo las mejores Rojo-1 x Rojo-2, Rojo-1 x Azul-1 y Rojo-1 x Azul-2; y para RAha las mejores cruzas fueron Rojo-1 x Rojo-2 y Rojo-1 x Azul-2.

Palabras clave: Zea mays, antocianinas, heterosis.

\section{SUMMARY}

The red, blue and purple maize (Zea mays L.) kernels contain anthocyanins of importance in the human diseases prevention. Nowadays, the selection of maize based on kernel color is considered relevant. To define efficient selection methodologies is convenient to apply genetics designs that allow analyzing the genetic contribution on the quantitative variation in the traits of interest. A genetic design was used to estimate general (GCA) and specific (SCA) combining abilities, maternal (ME) and reciprocal (RE) effects of the anthocyanin concentrations per unit of weight of grain $\left(\mathrm{AC}_{\mathrm{kg}}\right)$ and the anthocyanin yield per hectare (AYha). An experiment of diallel crosses between five native populations with different grain color was established where some agronomic traits were measured. In another experiment, $F_{2}$ seed was obtained using controlled pollination, to determine the concentrations of anthocyanin in the whole grain. Using data from both experiments the anthocyanins yield per plant (AY $Y_{\text {pta }}$ ), per hectare (AYha) and the heterosis (\%) were calculated. There was similarity among progenitors in the agronomic traits, but they differed on their capacity to accumulate anthocyanin. The GCA effects were superior in most of the agronomic traits compared to the SCA. The AY $Y_{\text {pta }}$ had significant effects of GCA, SCA and RE, being the Red-1 population the one of greater GCA. There was heterosis in crosses for $\mathrm{AC}_{\mathrm{kg}}$, where the best ones were Red-1 x Red-2, Red-1 x Blue-1 and Red-1 x Blue-2; and for $A Y_{\text {ha }}$ the best crosses were Red-1 x Red-2 and Red-1 x Blue-2.

Index words: Zea mays, anthocyanins, heterosis.

\section{INTRODUCCIÓN}

Una amplia variación genética en sus características morfológicas, fisiológicas y bioquímicas, aunadas a su adaptabilidad, han permitido la selección de maíces (Zea mays L.) para usos especiales. Por varias décadas, el objetivo primario de los programas de fitomejoramiento de maíz ha sido incrementar el rendimiento de grano; en lustros recientes se ha dado atención al desarrollo de variedades mejoradas que incorporen tanto composición como concentración de compuestos fenólicos, antocianinas, carotenoides, glucosinolatos y fitoestrógenos, relevantes en la preservación de la salud humana (Della Pena, 1999), aunado al hecho de que los fitoquímicos del tipo nutracéuticos presentan una demanda mundial creciente (Basu et al., 2007). El interés por el grano pigmentado de maíz se debe al contenido de varios compuestos de importancia nutracéutica, tales como 
antocianinas y ácidos fenólicos, que son antioxidantes y antimutagénicos (Pedreschi y Cisneros, 2006). Particularmente, el color del grano se ha seleccionado por los agricultores mesoamericanos durante la domesticación del maíz, lo que dio lugar a variantes en pigmentación (negro, púrpura, café, rojo, anaranjado, amarillo, blanco $\mathrm{y}$ variegados), que se usan en alimentos y en eventos especiales (Johanneseen, 1982).

Para que la selección sea eficaz, es conveniente que se base en la estimación de parámetros genéticos de las características de importancia, mediante el análisis estadístico de datos fenotípicos de las progenies segregantes, de los progenitores o de ambos. Existen diseños genéticos apropiados para hacer tales estimaciones (Suriyagoda y Peiris, 2007); al respecto, el esquema de cruzas dialélicas permite estudiar el control genético de características cuantitativas mediante la valoración de la aptitud combinatoria general (ACG) y específica (ACE), así como el análisis de la heterosis (Shattuck et al., 1993).

En maíces de grano púrpura, azul, rojo y blanco, es nula la información publicada sobre la aptitud combinatoria del contenido de antocianinas en el grano. Los estudios pioneros de la pigmentación del grano de maíz por antocianinas pretendieron determinar su herencia desde el enfoque mendeliano (East, 1912). Sin embargo, se encontró que algunos alelos de ciertos loci del color del grano segregan de manera impredecible (Coulter, 1920). Entre las razones por las cuales se presenta esa variación en el color de grano están los efectos maternos (Fujii y Kuwada, 1916), la influencia de transposones (Peterson, 2005), la ocurrencia de fenómenos epigenéticos y los cambios sutiles a nivel transcripcional de los genes estructurales de la ruta biosintética (epistasis transcripcional) (Springer y Stupar, 2007).

Este trabajo tuvo como objetivo estimar la aptitud combinatoria (ACG y ACE) del rendimiento de antocianinas y otras características de interés agronómico, en semilla de la generación $\mathrm{F}_{2}$ de las cruzas posibles (directas y recíprocas) entre poblaciones nativas contrastadas en color de grano, bajo las hipótesis de que los progenitores de grano de color rojo y azul manifiestan alta ACG, en lugar de alta ACE, y que hay efectos maternos sobre el contenido de pigmento en el grano.

\section{MATERIALES Y MÉTODOS}

\section{Sitio experimental y material genético}

Se estudió semilla de la generación $\mathrm{F}_{2}$ de las cruzas posibles (directas y recíprocas) entre cinco poblaciones nativas de maíz, representativas de grupos de color delimitados en el estudio de Espinosa et al. (2006), en donde se exploraron diferentes grados de coloración y contenido de antocianinas en el grano. Las poblaciones aquí usadas fueron denominadas Rojo-1 (del grupo Púrpura), Azul-1 (del grupo Azul), Azul-2 (del grupo Azul), Rojo-2 (del grupo Rojo) y Blanco (del grupo Blanco).

\section{Estudio agronómico}

Con la semilla $F_{1}$ de los cinco progenitores y las 20 cruzas posibles, se estableció un experimento el 12 de mayo de 2005 en Montecillo, Texcoco, Estado de México (19²9’ LN, 98 58', 2220 msnm). Se usó un diseño de bloques completos al azar con tres repeticiones, y la parcela consistió de dos surcos de $5 \mathrm{~m}$ de longitud con 21 plantas cada uno. El manejo agronómico se hizo de acuerdo con las recomendaciones del Instituto Nacional de Investigaciones Forestales, Agrícolas y Pecuarias para el cultivo de maíz en los Valles Altos Centrales de México.

De cada unidad experimental se tomaron los siguientes datos: días a floración masculina (DFM) y femenina (DFF), altura de la planta (ALP, en $\mathrm{cm}$ ), número de mazorcas por planta (NMzP), calculado con base en el cociente número de mazorcas cosechadas/número de plantas en competencia completa por parcela; número de hileras por mazorca $(\mathrm{NHMz})$, número de granos por hilera $(\mathrm{NGH})$, número de granos por mazorca $(\mathrm{NGMz}=$ $\mathrm{NHMz} \times \mathrm{NGH}$ ), peso individual del grano (PIG, estimado del peso de tres muestras de 500 granos por parcela), rendimiento de grano por planta $\left(\mathrm{RG}_{\mathrm{pta}}=\mathrm{NMzP} \times \mathrm{NGMz}\right.$ x PIG, en g) y el rendimiento de grano por hectárea ( $R_{\text {ha }}$ $=\mathrm{RG}_{\text {pta }} \mathrm{x}$ DEN, en $\mathrm{t} \mathrm{ha}^{-1}$ ) donde DEN fue la densidad de 50000 plantas hä ${ }^{-1}$.

\section{Estudio bioquímico}

Las cruzas y los progenitores se establecieron en un bloque completo sin repetición en dos ambientes. El primero se sembró el 12 de mayo de 2005 en un suelo franco arcilloso. El segundo se sembró el 27 de mayo del mismo año pero en suelo franco arenoso. En ambos ambientes las dimensiones de la unidad experimental fueron similares y en cada unidad experimental se hicieron manualmente de 15 a 20 polinizaciones fraternales, para presentar así la segregación de la generación $\mathrm{F}_{2}$ en semilla.

De cada parcela se obtuvo una muestra de grano, que se separó y contó por clases de color (tonalidades), dada la segregación observada en esta generación. Posteriormente se calcularon las frecuencias relativas (\%) de cada clase de grano. 
Para la cuantificación de las antocianinas se tomaron aleatoriamente tres muestras de $40 \mathrm{~g}$ de grano con $12 \%$ de humedad, de cada cruza y progenitor. Los granos se pulverizaron con un molino eléctrico tipo ciclón (Cyclone ${ }^{\circledR}$ Sampler Mill. UD Co. Colorado, USA), y la harina que pasó la malla de poros de $0.5 \mathrm{~mm}$ de diámetro se conservó en congelación $\left(<0{ }^{\circ} \mathrm{C}\right)$ en sobres de papel hasta su análisis.

Previo a la extracción de antocianinas, las muestras de harina se secaron en estufa a $60{ }^{\circ} \mathrm{C}$ durante $24 \mathrm{~h}$; de cada una se tomaron $5 \mathrm{~g}$ que se colocaron en un matraz Erlenmeyer de $125 \mathrm{~mL}$. Se agregaron $20 \mathrm{~mL}$ de solución de extracción (SE) compuesta de ácido trifluoracético 1.0 $\%$ (TFA, Sigma ${ }^{\circledR}$ ) en metanol grado reactivo (Baker $\left.{ }^{\circledR}\right)$, y se dejó en refrigeración durante $24 \mathrm{~h}$. Pasado este tiempo se midió el volumen del sobrenadante del cual se tomó una alícuota de $5 \mathrm{~mL}$ que se filtró en papel Wathman ${ }^{\circledR}$ No. 5; el filtrado se diluyó tres veces con la SE y se tomaron $3 \mathrm{~mL}$ para medir su absorbancia simultáneamente en celdas de cuarzo a $520 \mathrm{~nm}$ (Abs520) y $700 \mathrm{~nm}$ (Abs700) en un espectrofotómetro Perkin Elmer ${ }^{\circledR}$ modelo UV/Vis Lamda 21. Se hizo una segunda extracción con la adición de $20 \mathrm{~mL}$ de SE al residuo de harina en el mismo matraz. Para estimar el contenido total de antocianinas, a la absorbancia de cada muestra y de cada repetición se le sustrajo la absorbancia de la turbidez (Abs700) y la absorbancia del extracto del grano blanco (Absgrano blanco), para contrarrestar la interferencia de otros compuestos diferentes a las antocianinas, con la fórmula: Absajustada = Abs520 - Abs700 - Absgrano blanco. Con el valor de Absajustada se determinó el contenido total de antocianinas por unidad de volumen (AT, mg $\mathrm{L}^{-1}$ ), mediante la aplicación de una ecuación de regresión de una línea patrón [AT = (AbSajustada x 0.0636) - (0.0014), $\mathrm{R}^{2}=0.999$ ], calibrada con diferentes concentraciones de pelargonidina clorada $\left(\right.$ Sigma $\left.{ }^{\circledR}\right)$. El contenido de pigmento también se expresó por unidad de peso de grano completo $\left(\mathrm{CA}_{\mathrm{kg}}\right.$, en $\left.\mathrm{mg} \mathrm{kg}^{-1}\right)$ en cada extracción.

\section{Rendimiento de antocianinas por hectárea}

Con el promedio del $\mathrm{CA}_{\mathrm{kg}}$ se calculó el rendimiento de antocianinas por planta ( $\mathrm{RA}_{\text {pta, }}$ en $\mathrm{mg}$ ), con base en el $\mathrm{RG}_{\mathrm{pta}}$ estimado en el estudio agronómico con plantas sujetas a polinización libre: $\mathrm{RA}_{\mathrm{pta}}=\left(\mathrm{RG}_{\mathrm{pta}} \mathrm{x} \mathrm{CA}_{\mathrm{kg}}\right) /$ 1000. Con este valor se estimó el rendimiento potencial de antocianinas por unidad de superficie $\left(\mathrm{RA}_{\mathrm{ha}}, \mathrm{kg} \mathrm{ha}^{-1}\right)$, al considerar la densidad de plantas y al rendimiento del pigmento por planta, como sigue: $\mathrm{RAha}=\mathrm{RApta} \times \mathrm{DEN}$.

$\mathrm{La}$ heterosis respecto al progenitor medio $(\mathrm{He})$ y heterobeltiosis $(\mathrm{Hb})$ de cada cruza se calcularon con las fórmulas de Hochholdinger y de Hoecker (2007) en las variables $\mathrm{CA}_{\mathrm{kg}} \mathrm{y}$ RAha, de la siguiente manera:

$$
H e=\left(\text { Cruza }-\left[\frac{P i+P j}{2}\right]\right)(100) ; H b=\left(\text { Cruza }-P_{m}\right)(100)
$$

Donde $P i$ y $P j$ son los valores de los progenitores $i$ y $j$ de la cruza, Cruza indica el valor de la cruza simple de un par de progenitores, y $P_{m}$ es el valor del mejor progenitor.

\section{Análisis estadístico}

El análisis estadístico de las variables DFM, DFF, ALP, NMzP, NGMz, PIG, RGa y RApta, en el arreglo de cruzas dialélicas, se hizo con el algoritmo computacional desarrollado por Mastache y Martínez (2003) correspondiente al Modelo I y Método I de Griffing (1956).

La comparación estadística entre los valores de ACG y EM de los progenitores se hizo con base en la estimación de la varianza de esos parámetros. Para esto se calculó la diferencia significativa honesta (DSH) como lo cita Griffing (1956).

$$
\begin{gathered}
A C G_{D S H}=\left[q_{\alpha}(p, g l e)\right]\left[\sqrt{\left(\frac{p-1}{2 r p^{2}}\right)(C M E)}\right] ; \\
E M_{D S H}=\left[q_{\alpha}(p, g l e)\right]\left(\sqrt{\frac{C M E}{2 r}}\right)
\end{gathered}
$$

Donde $q_{\alpha}$ es el cuantil para la prueba de Tukey para comparar las medias de los $p$ progenitores, gle son los grados de libertad del error establecidos en el análisis de varianza del Modelo I y Método I, $r$ es el número de repeticiones, y $C M E$ es el cuadrado medio del error del análisis de varianza mencionado.

Se hizo un análisis de varianza y la prueba de medias de Tukey para $\mathrm{CA}_{\mathrm{kg}}$ y RAha de las cruzas, con un diseño completamente al azar con seis repeticiones y de bloques al azar con tres repeticiones, respectivamente, mediante el sistema $\mathrm{SAS}{ }^{\circledR}$ para Windows versión 9.0.

\section{RESULTADOS Y DISCUSIÓN}

\section{Características de los progenitores}

Sólo hubo diferencias $(\mathrm{P} \leq 0.05)$ entre los progenitores en las variables número de mazorcas por planta $(\mathrm{NMzP})$, peso individual del grano (PIG), rendimiento de antocianinas por planta y contenido de antocianinas por kilogramo de grano (Cuadro 1). A pesar de que hubo 
diferencias $(\mathrm{P} \leq 0.05)$ entre poblaciones en dos componentes morfológicos del rendimiento (NMzP y PIG), éstas no tuvieron impacto en el rendimiento de grano por hectárea. Se sabe que el rendimiento de grano depende en mayor magnitud del número de granos por planta y en menor magnitud del peso individual de grano (Maddoni y Otegui, 2006). Esto explica que al no haber diferencias en el número de granos por mazorca, el rendimiento de grano por hectárea tampoco varió significativamente. Se considera que el número máximo de granos por mazorca en híbridos de maíz para obtener un alto rendimiento de grano, oscila entre 600 y 700 (Ritchie y Alagarswamy, 2003). Las poblaciones nativas estudiadas presentaron valores aún bajos para este carácter, ya que fueron seleccionadas por su uniformidad en el porte de la planta, por el periodo de floración y por el color contrastado del grano (Espinosa et al., 2006), pero no necesariamente por su mayor rendimiento de grano. Con estos resultados se corrobora que la diferencia entre progenitores se centró en el color de grano.

En la población Rojo-1 se encontraron los valores más altos de rendimiento de antocianinas por planta y contenido del pigmento por kilogramo de grano (43.5 $\mathrm{mg} /$ planta y $377.0 \mathrm{mg} \mathrm{kg}^{-1}$, respectivamente). Se observó que la producción de antocianinas no presentó asociación con los valores de los componentes del rendimiento ni con las características fenológicas, lo que brinda la oportunidad de formar variedades e híbridos con características agronómicas aceptables, sin alterar la concentración del pigmento (Cuadro 1). A este respecto, Antonio et al. (2004) al evaluar poblaciones nativas de grano azul de la raza Chalqueño, encontraron entre ellas una amplia variabilidad fenotípica para características agronómicas, lo que hace posible practicar selección en programas de mejoramiento genético.

Cuadro 1. Características agronómicas, componentes del rendimiento y contenido de antocianinas en cinco poblaciones nativas de maíz estudiadas en el experimento dialélico. Montecillo, Texcoco, Edo. de México. 2005.

\begin{tabular}{lccccccccccc}
\hline Progenitor & DFM & DFF & ALP & NMzP & NHMz & NGH & NGMz & PIG & RGha & RApta & CAkg \\
\hline Rojo-1 & $80 \mathrm{a}$ & $83 \mathrm{a}$ & $210 \mathrm{a}$ & $1.6 \mathrm{a}$ & $12.7 \mathrm{a}$ & $22 \mathrm{a}$ & $277.0 \mathrm{a}$ & $253 \mathrm{a}$ & $5.7 \mathrm{a}$ & $43.5 \mathrm{a}$ & $376.9 \mathrm{a}$ \\
Rojo-2 & $82 \mathrm{a}$ & $86 \mathrm{a}$ & $203 \mathrm{a}$ & $1.1 \mathrm{~b}$ & $14.0 \mathrm{a}$ & $23 \mathrm{a}$ & $324.0 \mathrm{a}$ & $241 \mathrm{ab}$ & $4.5 \mathrm{a}$ & $12.0 \mathrm{c}$ & $131.5 \mathrm{c}$ \\
Azul-1 & $80 \mathrm{a}$ & $83 \mathrm{a}$ & $202 \mathrm{a}$ & $1.2 \mathrm{~b}$ & $14.4 \mathrm{a}$ & $21 \mathrm{a}$ & $306.5 \mathrm{a}$ & $225 \mathrm{ab}$ & $4.3 \mathrm{a}$ & $25.4 \mathrm{~b}$ & $292.3 \mathrm{ab}$ \\
Azul-2 & $83 \mathrm{a}$ & $90 \mathrm{a}$ & $207 \mathrm{a}$ & $1.3 \mathrm{ab}$ & $13.3 \mathrm{a}$ & $23 \mathrm{a}$ & $303.8 \mathrm{a}$ & $190 \mathrm{~b}$ & $4.0 \mathrm{a}$ & $16.6 \mathrm{bc}$ & $204.3 \mathrm{bc}$ \\
Blanco & $80 \mathrm{a}$ & $86 \mathrm{a}$ & $223 \mathrm{a}$ & $1.1 \mathrm{~b}$ & $14.4 \mathrm{a}$ & $22 \mathrm{a}$ & $324.4 \mathrm{a}$ & $259 \mathrm{a}$ & $4.9 \mathrm{a}$ & $0.0 \mathrm{~d}$ & $0.0 \mathrm{~d}$ \\
DSH & 6.6 & 10.7 & 36.6 & 0.364 & 2.7 & 5.1 & 55.4 & 56.8 & 2.0 & 9.5 & 5.4 \\
\hline
\end{tabular}

Valores con la misma letra en una columna son estadísticamente iguales (Tukey, 0.05); DSH = diferencia significativa honesta; DFM = días a floración masculina; DFF = días a floración femenina; ALP = altura de planta $(\mathrm{cm})$; NMzP = número de mazorcas por planta; $\mathrm{NHMz}=$ número de hileras por mazorca; $\mathrm{NGH}=$ número de granos por hilera; $\mathrm{NGMz}=$ número de granos por mazorca; PIG = peso individual del grano $(\mathrm{mg})$; $\mathrm{RGha}$ $=$ rendimiento de grano por hectárea $\left(\mathrm{t} \mathrm{ha}{ }^{-1}\right) ; \mathrm{RA}_{\mathrm{pta}}=$ rendimiento de antocianinas por planta $(\mathrm{mg} / \mathrm{planta}) ; \mathrm{CA} \mathrm{kg}=$ concentración de antocianinas en el grano completo ( $\mathrm{mg} \mathrm{kg}^{-1}$ de grano).

Cuadro 2. Cuadrados medios del análisis de varianza de las cruzas posibles entre cinco poblaciones nativas de maíz con diferentes grados de pigmentación. Evaluación de la generación F2. Montecillo, Texcoco, Edo. de México. 2005.

\begin{tabular}{|c|c|c|c|c|c|c|c|c|c|c|c|}
\hline $\mathrm{FV}$ & Gl & DFM & DFF & ALP & NMzP & NHMz & NGH & NGMz & PIG & RGha & RApta \\
\hline Bloques & 2 & $0.21 \mathrm{~ns}$ & $0.09 \mathrm{~ns}$ & $40.5 \mathrm{~ns}$ & $0.047 \mathrm{~ns}$ & $1.20 \mathrm{~ns}$ & $10.9 \mathrm{~ns}$ & $707.5 \mathrm{~ns}$ & $0.0005 \mathrm{~ns}$ & $1.1 \mathrm{~ns}$ & $14.57 \mathrm{~ns}$ \\
\hline Cruzas & 24 & $13.04^{* *}$ & $14.94^{* *}$ & $561.1^{* *}$ & $0.063^{* *}$ & $1.05^{*}$ & $12.9^{* *}$ & $3394.5^{* *}$ & $0.004^{* *}$ & $3.2^{* *}$ & $729.54^{* *}$ \\
\hline ACG & 4 & $33.49^{* *}$ & $28.81^{*}$ & $1266.4^{*}$ & $0.216^{* *}$ & $2.23^{*}$ & $25.8^{*}$ & $2993.1 \mathrm{~ns}$ & $0.012^{* *}$ & $6.3^{*}$ & $3075.78^{* *}$ \\
\hline $\mathrm{ACE}$ & 10 & $14.46^{* *}$ & $20.62^{* *}$ & $417.7 \mathrm{~ns}$ & $0.054^{* *}$ & $0.98 \mathrm{~ns}$ & $17.9^{* *}$ & $5580.2^{* *}$ & $0.003^{* *}$ & $4.5^{* *}$ & $264.67^{* *}$ \\
\hline ACG/ACE & & 2.30 & 1.40 & 3.0 & 4.000 & 2.20 & 1.4 & 0.5 & 4.000 & 1.4 & 11.60 \\
\hline EM & 4 & $2.10 \mathrm{~ns}$ & $3.55 \mathrm{~ns}$ & $628.8 \mathrm{~ns}$ & $0.007 \mathrm{~ns}$ & $0.97 \mathrm{~ns}$ & $3.0 \mathrm{~ns}$ & $1591.6 \mathrm{~ns}$ & $0.001 \mathrm{~ns}$ & $1.4 \mathrm{~ns}$ & $466.37 \mathrm{~ns}$ \\
\hline ER & 6 & $4.32 \mathrm{~ns}$ & $3.82 \mathrm{~ns}$ & $284.7 \mathrm{~ns}$ & $0.014 \mathrm{~ns}$ & $0.44 \mathrm{~ns}$ & $2.5 \mathrm{~ns}$ & $1221.1 \mathrm{~ns}$ & $0.0007 \mathrm{~ns}$ & $0.3 \mathrm{~ns}$ & $115.62^{* *}$ \\
\hline Error & 48 & 3.61 & 6.07 & 215.1 & 0.016 & 0.57 & 5.1 & 1348.8 & 0.001 & 1.1 & 29.09 \\
\hline $\mathrm{CV}(\%)$ & & 2.40 & 3.00 & 6.6 & 10.10 & 5.30 & 9.1 & 10.4 & 11.8 & 17.2 & 16.60 \\
\hline
\end{tabular}

$\mathrm{FV}$ = fuentes de variación; $\mathrm{Gl}=$ grados de libertad; $\mathrm{ACG}=$ aptitud combinatoria general; $\mathrm{ACE}=$ aptitud combinatoria específica; EM = efecto materno; $\mathrm{ER}=$ efecto recíproco; $\mathrm{DFM}=$ días a floración masculina; $\mathrm{DFF}=$ días a floración femenina; $\mathrm{ALP}=$ altura de planta; $\mathrm{NMzP}=$ número de mazorcas por planta; $\mathrm{NHMz}=$ número de hileras por mazorca; $\mathrm{NGH}=$ número de granos por hilera; $\mathrm{NGMz}=$ número de granos por mazorca; PIG $=$ peso individual del grano; $\mathrm{RG}_{\mathrm{ha}}=$ rendimiento de grano por ha; $\mathrm{RA}$ pta $=$ rendimiento de antocianinas por planta; ${ }^{*}=\mathrm{P} \leq 0.05 ;{ }^{* *}=\mathrm{P} \leq 0.01 ; \mathrm{ns}=$ no significativo. 


\section{Análisis dialélico}

Hubo diferencias $(\mathrm{P} \leq 0.01)$ entre las medias de las cruzas (directas y recíprocas) y de progenitores en cada una de las variables estudiadas (Cuadro 2). El efecto de ACG resultó significativo $(\mathrm{P} \leq 0.05)$ en todas las variables, excepto en el número de granos por mazorca. Además, los efectos de ACE fueron significativos ( $\mathrm{P} \leq$ $0.01)$ en todas las variables, con excepción del número de hileras por mazorca y de altura de planta. De acuerdo con Reif et al. (2007) y con base en el cociente entre los cuadrados medios de ACG/ACE, en casi todas las variables, con excepción del número de granos por mazorca, fue relevante la acción génica aditiva. En variedades de maíz, Jumbo y Carena (2008) encontraron efectos significativos de la ACG y de la interacción ACG $\mathrm{x}$ ambiente, sobre el rendimiento de grano por hectárea; sin embargo, los efectos de ACE, EM o ER no fueron significativos. En otro estudio con variedades de maíz, Mahto y Ganguli (2003) detectaron efectos significativos de la ACG y ACE para días a floración masculina y femenina, altura de planta, número de hileras por mazorca, número de granos por hilera y peso individual de grano; además, los cuadrados medios de la ACG fueron mayores que los de la ACE. Barrera et al. (2005) reportaron en su estudio de cruzas entre razas mejoradas de maíz que fueron significativos los efectos de ACG y ACE para días a floración masculina y femenina, y para altura de planta, pero no fueron significativas las interacciones ACG x ambiente y ACE x ambiente. Estos estudios indican que los efectos aditivos y no aditivos dependen de las variedades, del ambiente, de la interacción, y de las variables consideradas. El ER sólo fue significativo $(\mathrm{P} \leq 0.05)$ para el rendimiento de antocianinas por planta, lo que indican que la dirección de la cruza tuvo influencia en la producción del pigmento.

\section{Aptitud combinatoria general}

En todas las variables estudiadas hubo diferencias $(\mathrm{P} \leq$ 0.05 ) entre poblaciones en el efecto de ACG (Cuadro 3). En general, las poblaciones Rojo-1, Azul-2 y Blanco presentaron efectos estadísticamente similares para rendimiento de grano por hectárea. Sin embargo, sólo Rojo-1 resultó superior al resto en ACG para rendimiento de antocianinas por planta.

Cuando en un experimento dialélico se estudian variedades de polinización libre, como en esta investigación, un valor alto de ACG en alguna variedad indica una mayor frecuencia de genes que incrementan la expresión del carácter bajo estudio, además de una mayor desviación en términos de frecuencias génicas con respecto a la media de las poblaciones involucradas en el dialélico (Ferreira et al., 2004). En este sentido, la población Rojo-1 fue la mejor en producción de antocianinas por hectárea, característica de interés en este estudio.

\section{Heterosis en rendimiento de antocianinas}

El mejor progenitor $(\mathrm{P} \leq 0.05)$ para contenido de antocianinas por kilogramo de grano $\left(\mathrm{CA}_{\mathrm{kg}}\right)$ fue el Rojo-1 $\left(377.0 \mathrm{mg} \mathrm{kg}^{-1}\right)$ y fue igual $(\mathrm{P} \leq 0.05)$ a la mejor cruza Rojo-1 x Rojo-2 (Cuadro 4). Como se esperaba, el progenitor Blanco no tuvo antocianinas. Algunas cruzas presentaron valores positivos de heterosis, tal como Rojo1 x Rojo-2, lo que indica un efecto de dominancia completa del progenitor Rojo-1.

Cuadro 3. Efectos de ACG en variables agronómicas de cinco poblaciones nativas de maíz contrastadas en color de grano. Evaluación de la generación $F_{2}$, Montecillo, Texcoco, Edo. de México. 2005.

\begin{tabular}{|c|c|c|c|c|c|c|c|c|c|c|}
\hline Progenitor & Color & DFM & DFF & ALP & $\mathrm{NMzP}$ & NHMz & NGH & PIG & RGha & $\mathrm{RA}_{\text {pta }}$ \\
\hline Rojo-1 & & $-0.37 \mathrm{~b}$ & $-0.80 \mathrm{~b}$ & $-1.45 \mathrm{a}$ & $0.07 \mathrm{a}$ & $-0.40 \mathrm{~b}$ & $0.25 \mathrm{a}$ & $0.02 \mathrm{a}$ & $0.61 \mathrm{a}$ & $11.7 \mathrm{a}$ \\
\hline Rojo-2 & & $0.54 \mathrm{a}$ & $0.20 \mathrm{a}$ & $-2.44 b$ & $-0.05 b$ & $0.02 \mathrm{a}$ & $-0.15 \mathrm{a}$ & $0.01 \mathrm{a}$ & $-0.12 b$ & $-1.4 \mathrm{c}$ \\
\hline Azul-1 & & $-1.32 b$ & $-1.15 b$ & $-7.98 b$ & $-0.07 \mathrm{~b}$ & $0.38 \mathrm{a}$ & $-1.24 \mathrm{~b}$ & $-0.005 \mathrm{~b}$ & $-0.54 \mathrm{~b}$ & $2.4 \mathrm{~b}$ \\
\hline Azul-2 & & $1.34 \mathrm{a}$ & $1.44 \mathrm{a}$ & $4.18 \mathrm{a}$ & $0.11 \mathrm{a}$ & $-0.03 \mathrm{a}$ & $0.92 \mathrm{a}$ & $-0.03 \mathrm{c}$ & $0.03 \mathrm{a}$ & $1.7 \mathrm{~b}$ \\
\hline Blanco & & $-0.19 b$ & $0.31 \mathrm{a}$ & $7.70 \mathrm{a}$ & $-0.06 b$ & $0.03 \mathrm{a}$ & $0.02 \mathrm{a}$ & $0.01 \mathrm{a}$ & $0.02 \mathrm{a}$ & $-14.4 \mathrm{~d}$ \\
\hline DSH & & 1.25 & 1.62 & 9.67 & 0.08 & 0.50 & 1.50 & 0.02 & 0.68 & 3.6 \\
\hline
\end{tabular}

Valores con la misma letra en una columna son estadísticamente iguales (Griffing, 0.05); DSH = diferencia significativa honesta; $\mathrm{DFM}=$ días a floración masculina; $\mathrm{DFF}=$ días a floración femenina; $\mathrm{ALP}=$ altura de planta; $\mathrm{NMzP}=$ número de mazorcas por planta; $\mathrm{NHMz}=$ número de hileras de granos por mazorca; $\mathrm{NGH}=$ número de granos por hilera; PIG = peso individual del grano; $\mathrm{RG}_{\mathrm{ha}}=$ rendimiento de grano por hectárea; $\mathrm{RA}$ pta $=$ rendimiento de antocianinas por planta. 
Otras cruzas para el mismo caso fueron Rojo-1 x Azul2, Azul-2 x Azul-1 y Rojo-2 x Azul-2. Sin embargo, en algunas cruzas recíprocas se presentaron valores negativos de heterosis: Rojo-2 x Rojo-1, Azul-2 x Rojo-1, Azul-1 x Azul-2 y Azul-2 x Rojo-2. Las cruzas restantes presentaron valores bajos de heterosis y fueron aún más bajos en las cruzas recíprocas. Generalmente, las cruzas directas o recíprocas de los progenitores pigmentados (Rojo-1, Rojo-2, Azul-1 y Azul-2) con el progenitor Blanco presentaron una reducción significativa de $C A_{\mathrm{kg}}$, con relación a las cruzas entre los progenitores rojos y azules.

Respecto al rendimiento de antocianinas por hectárea ( $\left.R A_{h a}\right)$, el comportamiento heterótico fue alto $(\mathrm{P} \leq 0.05)$ en las cruzas Rojo-1 x Azul-2 (2.9 kg ha-1) y Rojo-1 x Rojo-2 (2.3 kg ha-1), que superaron al mejor progenitor
(Rojo-1) en 32.6 y $6.4 \%$, respectivamente. En la cruza Rojo-1 x Azul-2 la heterobeltiosis para RAha estuvo vinculada con un mayor rendimiento de grano por hectárea, pero no con una mayor concentración del pigmento por kilogramo de grano (Cuadro 4).

Las cruzas que también presentaron valores positivos de heterosis para $\mathrm{RA}$ ha, con respecto al progenitor medio, fueron: Rojo-1 x Azul-1, Azul-2 x Azul-1, Rojo-2 x Azul-2, Rojo-2 x Azul-1 y Blanco x Rojo-2. Las cruzas recíprocas que presentaron valores de heterosis positivos bajos y valores negativos fueron: Azul-1 x Rojo-1, Azul-1 x Azul-2, Azul-2 x Rojo-2, Azul-1 x Rojo-2, Rojo-2 x Blanco y Rojo-2 x Rojo-1. En general, todas las cruzas con el progenitor blanco presentaron $\mathrm{RA}_{\mathrm{ha}}$ superiores a $0.5 \mathrm{~kg} \mathrm{ha}^{-1}$.

Cuadro 4. Heterosis $(\mathrm{H}$, en \%) en la concentración por kilogramo $(\mathrm{CA} \mathrm{kg}$, en $\mathrm{mg})$ y rendimiento por hectárea (RAha, en $\mathrm{kg})$ de antocianinas, en poblaciones nativas de maíz contrastantes en color de grano y en sus cruzas posibles (directas y recíprocas). Montecillo, Texcoco, México. 2005.

\begin{tabular}{|c|c|c|c|c|c|c|}
\hline \multirow[b]{2}{*}{ Hembra } & \multirow[b]{2}{*}{ Variable } & \multicolumn{5}{|c|}{ Macho } \\
\hline & & Rojo-1 & Rojo-2 & Azul-1 & Azul-2 & Blanco \\
\hline \multirow[t]{4}{*}{ Rojo-1 } & $\mathrm{CA}_{\mathrm{kg}}$ & 377.0 & 383.6 & 265.2 & 339.3 & 54.9 \\
\hline & $\mathrm{CA}_{\mathrm{kg} H}$ & 0.0 & 50.9 & -20.8 & 33.4 & -70.9 \\
\hline & RAha & 2.2 & 2.3 & 1.7 & 2.9 & 0.4 \\
\hline & $\mathrm{RA}_{\mathrm{haH}}$ & 0.0 & 6.5 & 0.3 & 32.6 & -62.6 \\
\hline \multirow[t]{4}{*}{ Rojo-2 } & $\mathrm{CA}_{\mathrm{kg}}$ & 199.3 & 131.6 & 188.3 & 189.0 & 19.3 \\
\hline & $\mathrm{CA}_{\mathrm{kg} H}$ & -21.6 & 0.0 & -11.2 & 12.5 & -70.7 \\
\hline & RAha & 1.4 & 0.6 & 1.1 & 1.3 & 0.1 \\
\hline & $\mathrm{RAhaH}_{\mathrm{h}}$ & -2.6 & 0.0 & 18.6 & 84.9 & -59.2 \\
\hline \multirow[t]{4}{*}{ Azul-1 } & $\mathrm{CA}_{\mathrm{kg}}$ & 184.4 & 199.5 & 292.4 & 212.9 & 77.7 \\
\hline & $\mathrm{CA}_{\mathrm{kg} H}$ & -44.9 & -5.9 & 0.0 & -19.4 & -46.8 \\
\hline & RAha & 1.0 & 1.0 & 1.3 & 1.3 & 0.4 \\
\hline & $\mathrm{RA}_{\mathrm{haH}}$ & -41.4 & 7.6 & 0.0 & 19.6 & -31.7 \\
\hline \multirow[t]{4}{*}{ Azul-2 } & $\mathrm{CA}_{\mathrm{kg}}$ & 185.9 & 129.8 & 324.0 & 204.3 & 18.2 \\
\hline & $\mathrm{CA}_{\mathrm{kg} H}$ & -36.0 & -22.7 & 30.5 & 0.0 & -82.2 \\
\hline & RAha & 1.4 & 0.8 & 1.7 & 0.8 & 0.1 \\
\hline & $\mathrm{RA}_{\mathrm{haH}}$ & -5.6 & 15.6 & 61.4 & 0.0 & -74.1 \\
\hline \multirow[t]{4}{*}{ Blanco } & $\mathrm{CA}_{\mathrm{kg}}$ & 16.5 & 50.9 & 27.5 & 8.1 & 0.0 \\
\hline & $\mathrm{CA}_{\mathrm{kg} H}$ & -91.2 & -22.6 & -81.2 & -92.1 & 0.0 \\
\hline & RAha & 0.1 & 0.3 & 0.2 & 0.1 & 0.0 \\
\hline & $\mathrm{RAhaH}$ & -90.0 & 6.0 & -76.2 & -87.1 & 0.0 \\
\hline
\end{tabular}

$\mathrm{CA}_{\mathrm{kg} \text { DSH }}=145.16 ; \mathrm{RAha}_{\mathrm{DSH}}=0.520 ; \mathrm{DSH}=$ diferencia significativa honesta (Tukey, 0.05$)$. 
En un estudio genético de la acumulación de antocianinas en anteras de maíz, Hollick y Chandler (1998) cruzaron líneas contrastantes (colorida e incolora) en alelos del gen $P l$ (codificador de un factor de transcripción). Observaron segregación de pigmentación de las anteras en diferentes grados y en diferentes frecuencias relativas. En $10 \%$ de las plantas la intensidad de la pigmentación fue mayor que la del progenitor pigmentado, lo que indicó un efecto de sobredominancia y heterosis; es decir, hubo una mayor expresión del gen $P l$ en el heterocigoto. No obstante, el nivel de expresión no aumentó cuando se incrementó el número de alelos (dosis) en las células y no fue uniforme en todas las anteras evaluadas, por lo que los autores sugieren la ocurrencia de segregación de genes modificadores no identificados. Por su parte, Springer y Stupar (2007) indican que una mayor expresión fenotípica de pigmentación por antocianinas podría resultar de las interacciones epistáticas entre los factores de transcripción, y no por las interacciones entre los genes estructurales, es decir, epistasis transcripcional. Esto explicaría los valores positivos y negativos de la heterosis encontrada en el presente estudio en las cruzas entre las poblaciones nativas contrastantes en el color de grano y en el contenido de antocianinas.

Desde la perspectiva económica, el rendimiento de antocianinas por unidad de superficie también es importante, dado que el valor de este pigmento en grado alimenticio es de aproximadamente $\$ 1000.0$ USD por kilogramo (Cisneros, 2003). Ésta es una razón por la cual es importante generar estrategias para aumentar el rendimiento de este pigmento por medio del mejoramiento genético y a través del manejo de los factores ambientales controlables (Brown et al., 2003; Cisneros, 2003). Los resultados del presente estudio indican que un alto rendimiento de pigmento se puede lograr mediante heterobeltiosis en contenido de antocianinas por kilogramo de grano y en rendimiento del pigmento por hectárea, al usar progenitores contrastados en el contenido de antocianinas, las cuales se almacenan en el pericarpio y en la capa de aleurona.

\section{Segregación de tonalidades de color}

Los progenitores presentaron un solo color (Cuadro 3) pero la mayoría de cruzas segregaron de dos a cuatro tonalidades, con excepción de Azul-1 x Azul-2 y Azul-2 x Azul-1 (Cuadro 5) donde dominó (100 \%) el color del progenitor materno. Las cruzas Azul-1 x Blanco y Blanco $\mathrm{x}$ Azul-1 segregaron dos tonalidades, y las otras cruzas (Rojo-1 x Azul-1, Azul-1 x Rojo-1, Azul-1 x Rojo-2, Rojo-2 x Azul-1, Azul-2 x Rojo-2 y Rojo-2 x Azul-2) tres tonalidades, independientemente de la dirección de los cruzamientos (directa o recíproca). Por el contrario, dos cruzas (Rojo-1 x Rojo-2 y Azul-2 x Blanco) segregaron dos tonalidades en el sentido directo $\mathrm{y}$ tres en el recíproco; otras cruzas (Rojo-1 x Azul-2, Rojo-1 x Blanco y Rojo-2 x Blanco) presentaron tres tonalidades en las directas y cuatro en las recíprocas.

Algunas cruzas directas presentaron valores positivos de heterosis en contenido de antocianinas por kilogramo de grano, mientras que sus recíprocas presentaron valores negativos (Cuadro 4). Esto estuvo relacionado con la segregación de granos de menor intensidad de color en las cruzas recíprocas, en comparación con las directas, como en el caso de Rojo-1 x Azul-2, Rojo-2 x Rojo-1 y Azul-2 $x$ Rojo-2. La cruza de mayor contenido de pigmento fue Rojo-1 x Rojo-2, la cual presentó 83 \% de granos con una tonalidad similar al progenitor Rojo-1. Sin embargo, esto no ocurrió en las cruzas Azul-1 x Azul-2 y Azul-2 x Azul-1 donde la tonalidad fue similar entre cruzas, pero sí difirieron en el contenido de pigmento; esto sugiere que el abatimiento en la capacidad de biosíntesis observada en las cruzas se debió a cambios en la capacidad de biosíntesis en la capa de aleurona de los granos azules y que no influyeron en la tonalidad.

Fujii y Kuwada (1916) consideraron la constitución genética triploide (3n) del endospermo para comprender la herencia de la pigmentación. De este modo, la capa de aleurona de los granos Azules presenta el genotipo $A A A$, que en el Blanco es $a a a$, tal que en la $\mathrm{F}_{2}$ se manifiestan cuatro clases de cigotos, cuya constitución genética corresponde a $A A A, A A a, A a a$ y $a a a$. Si se supone que cada $A$ representa una unidad de intensidad del color azul o rojo, entonces en la segregación se genera un gradiente de color del grano, sobre todo si el número de loci se incrementa. Por otra parte, desde un enfoque mendeliano, los trabajos pioneros sobre los pigmentos del maíz encontraron que los factores (genes) responsables del color rojo o púrpura de la aleurona eran dominantes sobre el blanco (Weatherwax, 1919) y la aleurona púrpura presentaba xenia (efecto del progenitor macho) sobre el color rojo (Snyder, 1946). En este sentido, la diferencia entre las cruzas directas con respecto a las recíprocas en la tonalidad del color indicaría un efecto genético del progenitor materno. Sin embargo, la diferencia entre ambas cruzas puede obedecer a efectos epigenéticos, como la impresión alélica que ocurre comúnmente con algunos alelos de los factores de transcripción de la ruta de biosíntesis de las antocianinas en el grano de maíz (Sokolov, 2006). 
Cuadro 5. Clasificación de granos $F_{2}$ de cruzas dialélicas entre poblaciones nativas de maíz, con base en la tonalidad del color (escala visual de imagen: I = mayor, IV = menor) y frecuencia (\%) en cada cruza. Montecillo, Texcoco, Edo. de México. 2005.

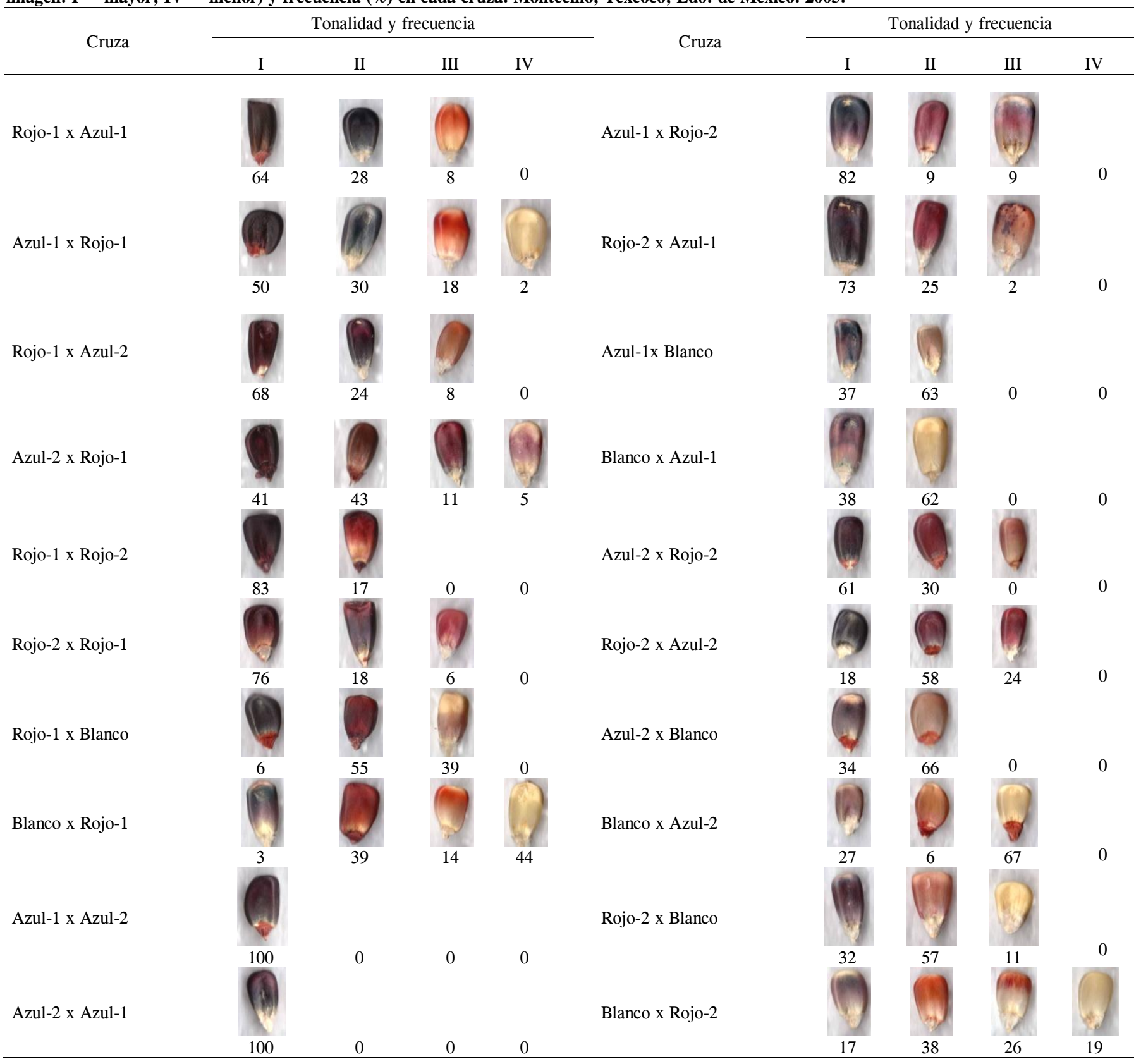

\section{CONCLUSIONES}

Con el estudio dialélico de cinco poblaciones nativas de maíz contrastantes en color de grano, se determinó un efecto significativo de la aptitud combinatoria general (ACG) en la mayoría de las características agronómicas estudiadas, y un efecto significativo de ACG, de aptitud combinatoria específica y de efecto recíproco sobre el rendimiento de antocianinas por planta. En esta última característica, el mejor progenitor fue el Rojo-1, pues presentó los valores altos y positivos de ACG.
El efecto materno no fue significativo, pero entre cruzas hubo diferencias en el contenido de antocianinas en el grano, donde sobresalieron las cruzas Rojo-1 x Rojo-2 y Rojo-1 x Azul-2. Este efecto positivo resultó del efecto significativo de heterobeltiosis y del de heterosis en varias cruzas directas.

Las cruzas de mayor contenido de antocianinas por kilogramo de grano no necesariamente fueron las mejores en el rendimiento del pigmento por hectárea. Esto indica que el mejoramiento genético debe considerar como 
criterio de selección una mayor concentración de antocianinas por grano (ubicadas en el pericarpio y en la capa de aleurona) y también un mayor número de granos por unidad de superficie.

Todas las cruzas que incluyeron el progenitor Blanco mostraron bajos rendimientos de antocianinas, de manera que la segregación de granos incoloros o de baja tonalidad se asoció con escasa acumulación del pigmento.

\section{BIBLIOGRAFÍA}

Antonio M M, J L Arellano V, G García de los S, S Miranda C, J A Mejía C, F V González C (2004) Variedades criollas de maíz azul raza chalqueño. Características agronómicas y calidad de semilla. Rev. Fitotec. Mex. 27:9-15.

Barrera G E, A Muñoz O, F Márquez S, A Martínez G (2005) Aptitud combinatoria en razas de maíz mejoradas por retrocruza limitada. I: Caracteres agronómicos. Rev. Fitotec. Mex. 28:231-242.

Basu S K, J E Thomas, S N Acharya (2007) Prospects for growth in global nutraceutical and functional food markets: a Canadian perspective. Austr. J. Basic Appl. Sci. 1:637-649.

Brown C R, R Wrolstad, R Durst, C P Yang, B Clevidence (2003) Breeding studies in potatoes containing high concentrations of anthocyanins. Amer. J. Potato Res. 80:241-249.

Cisneros Z L (2003) The use of controlled postharvest abiotic stresses as a tool for enhancing the nutraceutical content and adding value of fresh fruits and vegetables. J. Food Sci. 68:1560-1565.

Coulter M C (1920) Inheritance of aleurone color in maize. Bot. Gazette 69:407-425.

Della Penna D (1999) Nutritional genomics: manipulating plant micronutrients to improve human health. Science 285:375-379.

East E M (1912) Inheritance of color in the aleurone cells of maize. Amer. Naturalist 46:363-365.

Espinosa T E, Ma del C Mendoza C, F Castillo G (2006) Diversidad fenotípica entre poblaciones de maíz con diferentes grados de pigmentación. Rev. Fitotec. Mex. 29:19-23.

Ferreira F M, J I R Júnior, C A P Pacheco, C H O Silva and S M Filho Jr (2004) Genetic components of combining ability in a complete diallel. Crop Breed. App. Biotechnol. 4:338-343.

Fujii K, Y Kuwada (1916) On the comparison of factorial formula for zygotes in the study of inheritance of seed characters of Zea mays L. with notes on seed pigments. Bot. Magazine 30:83-88.
Griffing B (1956) A generalized treatment of the use of diallel crosses in quantitative inheritance. Heredity 10:31-50.

Hochholdinger F, N Hoecker (2007) Towards the molecular basis of heterosis. Trends Plant Sci. 12:1360-1385.

Hollick J B, V L Chandler (1998) Epigenetic allelic states of a maize transcriptional regulatory locus exhibit overdominant gene action. Genetics 150:891-897.

Johannessen C L (1982) Domestication process of maize continues in Guatemala. Econ. Bot. 36:84-99.

Jumbo M B, M J Carena (2008) Combining ability, maternal, and reciprocal effects of elite early maturing maize population hybrids. Euphytica 162:325-333.

Maddonni G A, M E Otegui (2006) Intra-specific competition in maize: contribution of extreme plant hierarchies to grain yield, grain yield components and kernel composition. Field Crops Res. 97:155-166.

Mahto R N, D K Ganguli (2003) Combining ability analysis in inter varietal crosses of maize (Zea mays L.). Madras Agric. J. 90:29-33.

Mastache L A A, A Martínez G (2003) Un algoritmo para el análisis, estimación y predicción de experimentos dialélicos balanceados. Rev. Fitotec. Mex. 26:191-200

Pedreschi R, L Cisneros Z (2006) Antimutagenic and antioxidant properties of phenolic fractions from Andean purple corn (Zea mays L.). J. Agric. Food Chem. 54:4557-4567.

Peterson P A (2005) The plant genetics discovery of the century: transposable elements in maize. Early beginnings to 1990 . Maydica 50:321-338.

Reif J C, F M Gumpert, S Fischer, A E Melchinger (2007) Impact of interpopulation divergence on additive and dominance variance in hybrid populations. Genetics 176:1931-1934.

Ritchie J T, G Alagarswamy (2003) Model concepts to express genetic differences in maize yield components. Agron. J. 95:4-9.

Shattuck V I, B Christie, C Corso (1993) Principles for Griffing's combining ability analysis. Genetics 90:73-77.

Snyder L H (1946) The Principles of Heredity. D. C. Heath and Company. Boston, USA. $450 \mathrm{p}$

Sokolov V A (2006) Imprinting in plants. Russian J. Gen. 42:10431052 .

Springer N M, R M Stupar (2007) Allelic variation and heterosis in maize: how do two halves make more than a whole? Genome Res. 17:264-275.

Suriyagoda L D B, B L Peiris (2007) Estimating genetic parameters from diallel experiments using SAS/IML. Sri Lankan J. Appl. Stat. 8:83-94.

Weatherwax P (1919) Gametogenesis and fecundation in Zea mays as the basis of xenia and heredity in the endosperm. Bull. Torrey Bot. Club 46:73-90. 